\title{
Neonate with Clustered Pustules on an Erythematous Base, A Diagnostic Dilemma
}

\author{
Jaclyn Otero, Zachary Gohsman, Kendall Steadmon and Molly Posa* \\ Department of Pediatrics, University of Florida, USA
}

*Corresponding author: Molly Posa, Department of Pediatrics, University of Florida, USA.

Received Date: : June 19, 2019

Published Date: June 21, 2019

\section{Background}

Pustules on an erythematous base can be a diagnostic dilemma in newborns ranging from a benign self-limited rash to a potentially life-threatening diagnosis, HSV. Timely assessment and identification are necessary because HSV infection requires lumbar puncture, antiviral treatment and brain imaging. In our case, strong clinical suspicion of an atypical presentation of erythema toxicum, followed by punch biopsy confirmation allowed for rapid diagnosis of this neonate.

\section{Case Presentation}

20 hour-old female neonates born at $39 \mathrm{w} 0 \mathrm{~d}$ via repeat C-section to a 30-year-old G2P1001 GBS negative, serology negative mother with a remote history of HSV 2 (no current or recently active lesions and not on prophylactic acyclovir during pregnancy). Delivery was uneventful and the neonate required only tactile stimulation for resuscitation and had assigned Apgars of 9 at 1 and 5 minutes. The mother received pitocin during delivery. At the time of the initial physical exam by the newborn nursery team, the neonate had normal vital signs and physical exam, appropriate number of voids and stools and was breastfeeding well. However, the nursery team was called to bedside on day of life 1 , because the infant was noted to have developed multiple areas of coalescing yellow vesicular lesions with erythematous bases on her right lateral hip, buttocks, and left inner thigh (Figure 1) but continued to be well appearing with normal vital signs and oral intake.

\section{Diagnosis and Treatment}

We consulted pediatric dermatology as there was concern for an atypical presentation for erythema toxicum versus HSV. They recommended a punch biopsy, which was performed on the cluster of lesions on the right lateral thigh (Figure 1). Biopsy demonstrated intraepidermal eosinophilic pustules and prominent dermal eosinophilic infiltrate, consistent with erythema toxicum neonatorum. No further treatment was required once the diagnosis of erythema was confirmed and the family was provided with anticipatory guidance.

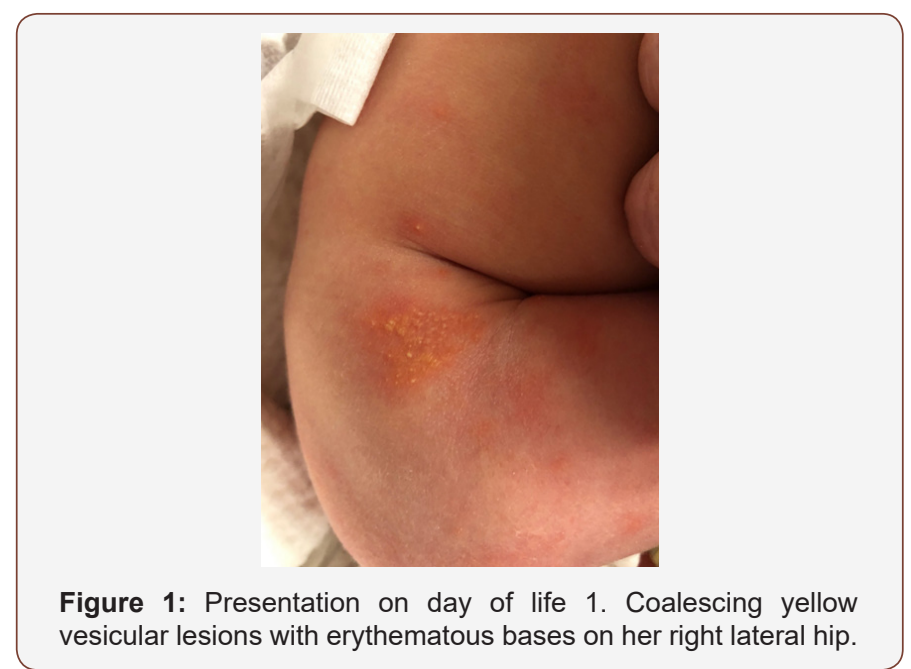

Patient Outcome

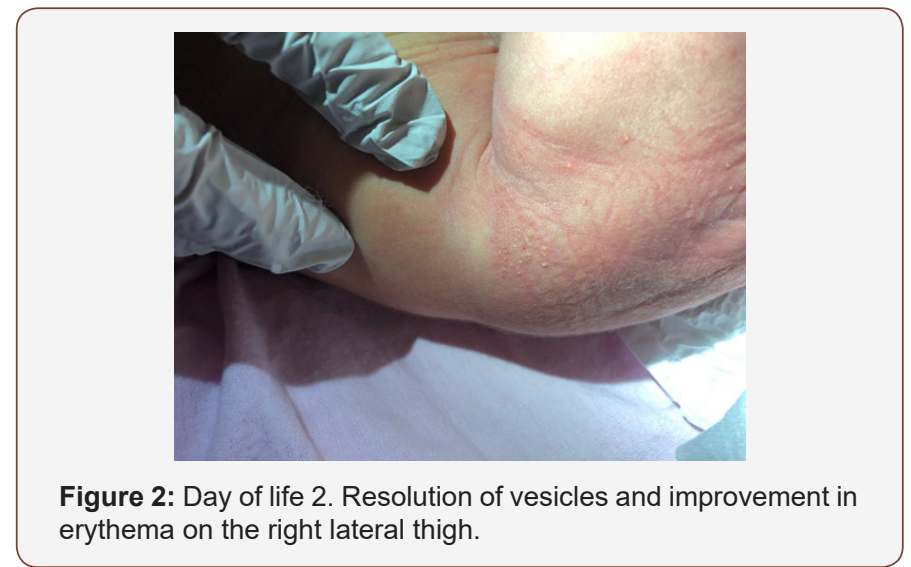

The neonate was discharged from the nursery on day two of life and the lesions were noted to have started to improve (Figure 2). Lesions had completely resolved by 2 weeks of life at follow-up with her pediatrician. 


\section{Discussion}

Given the presentation was most concerning for HSV versus erythema toxicum, our discussion will be limited to these diagnoses.

Our patient was diagnosed with erythema toxicum, a common, non-infectious dermatologic condition seen in newborns. Erythema toxicum neonatorum (ETN) is a common pustular disorder occurring in in the first 72 hours of life in approximately 23 percent of neonates [1]. ETN typically presents with multiple erythematous macules and papules, 1 to $3 \mathrm{~mm}$ in diameter, that progress to pustules on an erythematous base [2]. The lesions spare the palms and soles; they are usually distributed over the trunk and proximal extremities. The rash generally resolves in five to seven days, although it can wax and wane before complete resolution [2]. The diagnosis of ETN can almost always be made solely based on the clinical appearance of the rash. In difficult cases or atypical presentations, ETN can be confirmed by microscopic examination of a Wright-stained smear of the contents of a pustule, which demonstrates numerous eosinophils and occasional neutrophils [1].

The most concerning differential diagnosis for our patient was neonatal herpes (NHSV). Neonatal HSV infection is rare but life threatening. It occurs before 42 days of age and primarily results from intrapartum exposure to maternal cervical or vaginal lesions or by an ascending infection, often through apparently intact membranes [3]. Postnatal inoculation also may occur but is much less common [4]. NHSV symptoms frequently develop within days to four weeks after birth. HSV infection in newborns usually develops in one of three patterns: 1. Fulminant, disseminated disease involving multiple organs, 2. Localized to the skin, eyes, and mouth (presenting parts), 3. Localized central nervous system (CNS) disease [4]. Although NHSV infection can present with nonspecific symptoms, such as fever, poor feeding and decreased activity, skin lesions occur in most patients and may be present in all three of the above described patterns [4]. The skin lesions typically consist of 2 to $3 \mathrm{~mm}$ vesicles that may develop into pustules, crusts and erosions. They usually occur on the presenting part at delivery such as on the scalp, occasionally associated with placement of a fetal monitor electrode, or the face. Lesions also may occur on the trunk or buttocks (more commonly with a breech presentation) [4]. Like ETN, lesions are not commonly present at birth but develop later than ETN, around 6 to 13 days of life [5]. Infants with lesions at birth (congenital HSV infection) have intrauterine, rather than perinatal, infection [6].

Thus, with NHSV, one can usually diagnose and distinguish the difference from ETN on clinical grounds. First, herpes simplex lesions typically appear later than ETN, usually 6 to 13 days of age. Second, lesions are likely to be somewhat larger and in coalescing clusters. Third, neonates may have a fever and/or may appear clinically ill with NHSV. Finally, history of maternal HSV infection, particularly a primary infection around the time of delivery, may be noted in neonates with NHSV infection. However, most cases occur without history of infection. Given the overlap in the appearance of the lesions, caution is advised until NHSV can be ruled out. When there is strong suspicion of NHSV infection, a full sepsis workup should be initiated immediately and treatment with high-dose acyclovir begun pending confirmation of the diagnosis [7].

On the other hand, in less straight forward cases as ours, beyond the reasonable goal of avoiding an unwarranted invasive, costly work-up and unneeded medication use, the risks of even short courses of acyclovir must also be considered. Extravasation can lead to injury particularly in neonates. Additionally, the nephrotoxic side effects of acyclovir, thought to be caused by deposition of acyclovir crystals in the kidney, can occur with even brief treatment in neonates [8].

This leads to a diagnostics dilemma of not wanting to miss a serious illness while also not wanting to cause any patient harm from possible iatrogenic complications. Due to NHSV infection being associated with high morbidity and mortality, rapid diagnosis is crucial. In most medical center settings, a polymerase chain reaction (PCR) can be used to rapidly assess for herpes simplex virus. However, a negative PCR does not rule out HSV [9]. Typically, PCR results will return within 48 hours at our institution but can be delayed on a weekend. If PCR is not available, the laboratory diagnosis of neonatal HSV infection may be established through isolation of HSV in traditional or enhanced viral culture, which takes more time. Rapid direct immunofluorescence assays (DFA) may also be used for detection of viral antigens but can be difficult to obtain accurately. Infant serology is generally not helpful in the diagnosis of NHSV at the time of presentation as transplacental immunoglobulin G (IgG) antibodies cannot be differentiated from IgG produced by the infant $[9,10]$.

\section{Learning points}

1) Erythema toxicum and NHSV can both present with pustules on an erythematous base in a neonate.

2) Whether to test for HSV is often based on clinical picture, but there are benign mimickers that clinically appear similar.

3) There are limitations to HSV testing and which test to order may vary with presentation. Rapid diagnosis is critical.

\section{Acknowledgments}

None.

\section{Conflicts of interest}

No conflict of interest.

\section{References}

1. Berg FJ, Solomon LM (1987) Erythema neonatorum toxicum. Arch Dis Child 62(4):327-378.

2. Katsambas AD, Katoulis AC, Stavropoulos P (1999) Acne neonatorum: a study of 22 cases. Int J Dermatol 38(2):128-130.

3. Manice CS, Planet PJ, Chase HS, Lauren CT (2018) Management of afebrile neonates with pustules and vesicles in a pediatric emergency department. Pediatr Dermatol 35(5):660-665.

4. James SH, Kimberlin DW (2015) Neonatal Herpes Simplex Virus Infection. Infect Dis Clin North Am 29(3):391-400. 
5. Hoath SB, Narendran V (2011) The skin. In: Neonatal-Perinatal Medicine, ( $9^{\text {th }}$ edn), Fanaroff AA, Martin RJ, Walsh MC (Edts), Elsevier Mosby, St Louis, p.1705.

6. Erin Mathes, Renee M Howard (2018) Vesicular, pustular, and bullous lesions in the newborn and infant. In Rosamaria Corona (Edn)

7. Brien, James H (2010) 2-day-old neonatal patient with rash. Infectious Diseases in Children.

8. Brower L, Schondelmeyer A, Wilson P, Shah SS (2016) Testing and Empiric Treatment for Neonatal Herpes Simplex Virus: Challenges and
Opportunities for Improving the Value of Care. Hosp Pediatr 6(2): 108111.

9. Gail J Demmler-Harrison (2018) Neonatal herpes simplex virus infection: Clinical features and diagnosis. In Carrie Armsby (Edn)

10. Allen UD, Robinson JL (2014) Prevention and management of neonatal herpes simplex virus infections. Paediatr Child Health 19(4): 201-206. 